\title{
BMJ Open Total glucosides of paeony for rheumatoid arthritis: a protocol for a systematic review
}

Jing Luo, ${ }^{1}$ Di-Er Jin, ${ }^{1}$ Guo-Yan Yang, ${ }^{2}$ Ying-Ze Zhang, ${ }^{1}$ Jian-Ming Wang, ${ }^{1}$ Wei-Ping Kong, ${ }^{1}$ Qing-Wen Tao ${ }^{1}$

To cite: Luo J, Jin D-E, Yang G-Y, et al. Total glucosides of paeony for rheumatoid arthritis: a protocol for a systematic review. BMJ Open 2016;6 e010116. doi:10.1136/ bmjopen-2015-010116

- Prepublication history and additional material is available. To view please visit the journal (http://dx.doi.org/ 10.1136/bmjopen-2015010116).

Received 26 September 2015 Revised 18 February 2016 Accepted 23 February 2016

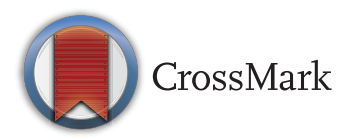

${ }^{1}$ Traditional Chinese Medicine Department of Rheumatism, China-Japan Friendship Hospital, Beijing, China ${ }^{2}$ National Institute of Complementary Medicine, Western Sydney University, Sydney, Australia

Correspondence to Professor Qing-Wen Tao; taogg1@sina.com

\section{ABSTRACT}

Introduction: Total glucosides of paeony (TGP) is a natural plant extract, which is widely used in China for treating rheumatoid arthritis (RA). Many relevant randomised controlled trials (RCTs) of TGP for RA are available, but they have not been systematically reviewed. This systematic review aims to examine the effectiveness and safety of TGP in patients with RA.

Methods and analyses: We will search for RCTs of TGP in the treatment of RA, performed up until February 2016, in PubMed, Embase, Cochrane Central Register of Controlled Trials, and four Chinese databases (Chinese Biomedical Database, China National Knowledge Infrastructure, Wanfang Database and Chinese Scientific Journal Database). Trial registers and reference lists of retrieved articles will also be searched to identify potential articles. RCTs comparing TGP with placebo, no treatment, or disease-modifying antirheumatic drugs for patients with RA will be retrieved. The primary outcomes will be disease improvement and disease remission. The secondary outcomes will be surrogate outcomes, symptoms, adverse effects, and quality of life. Two reviewers will independently extract data on participants, interventions, comparisons, outcomes, etc. The methodological quality of each included study will be evaluated using the Cochrane risk of bias tool, and the strength of evidence on prespecified outcomes will be assessed in accordance with the GRADE (Grading of Recommendations Assessment, Development and Evaluation) approach. Review Manager 5.3 software will be used for data analyses. Meta-analyses will be performed if the data are sufficiently homogeneous, both statistically and clinically. Possible publication bias will also be checked using funnel plots once the number of included studies is sufficient.

Ethics and dissemination: Ethics approval is not required, as this study will not involve patients. The results of this study will be submitted to a peerreviewed journal for publication, to inform both clinical practice and further research.

Trial registration number: CRD42015026345.

\section{INTRODUCTION}

\section{Description of the condition}

Rheumatoid arthritis (RA) is the most common type of chronic autoimmune

\section{Strengths and limitations of this study}

- This will be the first PRISMA (Preferred Reporting Items for Systematic Reviews and Meta-analyses)-compliant systematic review to assess the effectiveness and safety of total glucosides of paeony for patients with rheumatoid arthritis. It will provide a high-quality synthesis of current evidence for patients and rheumatologists seeking alternative and beneficial treatments for rheumatoid arthritis.

- In addition to measuring the methodological quality of included studies using the Cochrane risk of bias tool, this systematic review will evaluate the strength of evidence according to the GRADE approach, to inform clinical decision-makers.

- Some unpublished randomised controlled trials with negative findings might be missed, so funnel plots will be conducted to detect possible publication bias in order to obtain an objective conclusion.

arthritis that causes pain, stiffness, joint swelling, deformity and loss of function. Over 1.3 million Americans have been estimated to have RA, with a global prevalence of $0.24 \% .^{12}$ Data from the Global Burden of Disease 2010 Study show that disability-adjusted life years for RA increased from 3.3 million in 1990 to 4.8 million in $2010 .^{2}$ Therefore, RA remains a serious disease imposing a considerable burden on patients, their families and society. In order to relieve pain and avoid irreversible joint destruction and disability, RA requires early and systematic treatment with timely adjustment.

Disease-modifying antirheumatic drugs (DMARDs), with effects of lowering disease activity and retarding joint erosion, remain the first-line treatment for RA, but the most common concern about DMARDs is safety. A clinical trial published in the Annals of the Rheumatic Diseases reported that methotrexate and leflunomide are associated with 
increased incidence of hepatotoxicity. ${ }^{3}$ Some patients have to stop treatment with DMARDs because of adverse effects. In addition, some patients with RA do not respond to DMARDs. Biological agents, also known as biological DMARDs, have been proven to be effective for RA, especially for patients who fail to respond to DMARDs. ${ }^{45}$ However, the very high cost of biological agents limits their application. Furthermore, exposure to biological agents appears to confer an increased risk of serious infection. ${ }^{6}$ In such cases, natural products with therapeutic potential have drawn increased attention. ${ }^{7}$

\section{Description of the intervention}

Total glucosides of paeony (TGP) is an active compound extracted from the roots of a Chinese herb, Paeonia lactiflora Pallas, with paeoniflorin accounting for $90 \%$ of its active components. ${ }^{8}$ In China, TGP has been approved as a disease-modifying oral drug for RA since 1998 by the China Food and Drug Administration and is now widely used to treat RA. Many experimental studies have shown the anti-inflammatory and immunoregulatory actions of TGP. ${ }^{9-11}$ For example, a study investigating the effects of TGP on synoviocyte activity in rats with collagen-induced arthritis found that TGP could significantly decrease the production of tumour necrosis factor $\alpha$ and interleukin 1 and inhibit the proliferation of synoviocytes. ${ }^{9}$ Another study revealed that TGP treatment could significantly increase the number and percentage of Treg cells in lupus CD4+ T cells. ${ }^{10} \mathrm{~A}$ review published in 2011 reported that paeoniflorin had immunosuppressive effects in rats with adjuvant arthritis. ${ }^{11}$ In addition, beneficial effects of TGP have been reported in some clinical trials including randomised controlled trials (RCTs). ${ }^{12} 13$ A multicentre RCT including 370 patients with RA found that TGP might be effective in improving joint function without severe adverse effects. ${ }^{12}$ A recent RCT published in English which enrolled 268 patients with active RA suggested that TGP could significantly reduce the incidence of liver damage caused by methotrexate and leflunomide. $^{13}$

\section{Why it is important to perform this review}

TGP is a natural plant extract and is widely used to treat RA in China. Although four systematic reviews on TGP in RA treatment are available, ${ }^{14-17}$ all are in Chinese and none are adequate with respect to systematic reviewing according to the Preferred Reporting Items for Systematic Reviews and Meta-analyses (PRISMA) statement. ${ }^{18}$ Two reviews ${ }^{14} 15$ included seven studies (published in 20052007) and 10 studies (published in 2002-2010), respectively, with different methodological quality. However, no subgroup or sensitivity analysis was performed. A review including nine studies between 2005 and 2009 pooled different outcomes using a fixed-effects model. ${ }^{16}$ The latest review including 15 studies between 2005 and 2011 evaluated a composite outcome measure designated 'overall effect' as the main outcome measure. ${ }^{17}$ Owing to these shortcomings, the effect of TGP for RA has not be been adequately assessed. Therefore, a comprehensive, updated and PRISMA-compliant systematic review of RCTs is necessary to evaluate the effect of TGP for RA, to inform both clinical practice and further research.

\section{OBJECTIVES}

The objective of this systematic review is to assess the effectiveness and safety of TGP for patients with RA.

\section{METHODS AND ANALYSIS \\ Study registration}

The original protocol of this systematic review has been documented in PROSPERO (ID=CRD42015026345).$^{19}$ This is a revised version which will also be uploaded to PROSPERO. ${ }^{19}$ The systematic review will be conducted according to the revised protocol and reported in accordance with the PRISMA statement.

\section{Eligibility criteria}

Types of study

Only RCTs will be eligible for inclusion irrespective of language or publication status. Quasi-randomised trials will be excluded.

\section{Types of participant}

Adult participants (18 years and older) of any gender or ethnicity, meeting one recognised diagnostic criterion of RA (the 1987 American College of Rheumatology (ACR) criteria or the 2010 ACR/European League Against Rheumatism (EULAR) criteria) will be included. ${ }^{20}{ }^{21}$ Studies without description of diagnostic criteria will not be considered.

\section{Types of intervention}

Studies assessing TGP with or without co-intervention(s) for patients with RA regardless of dosage will be included. Control interventions should be placebo, no treatment, or DMARDs (traditional or biological). TGP compared with any Chinese patent medicine or herbal formulation will be ineligible. Complex intervention involving TGP but no separate report on outcomes of TGP will be excluded. The duration of therapy should be more than 12 weeks.

\section{Types of outcome measure}

\section{Primary outcomes}

1. Disease improvement (measured by any validated improvement criteria of RA, such as the ACR20 response $^{22}$ )

2. Disease remission (measured by any validated remission criteria of RA, such as the Disease Activity Score in 28 joints less than $2.6^{2324}$ )

\section{Secondary outcomes}

1. Adverse effects

2. Pain (measured by a visual analogue scale) 
3. Health-related quality of life (measured by a validated tool)

4. C-reactive protein

5. Erythrocyte sedimentation rate

\section{Search methods}

Electronic searches

The following databases will be searched from their inception to February 2016: PubMed, Embase, Cochrane Central Register of Controlled Trials, Chinese Biomedical Database (CBM), China National Knowledge Infrastructure (CNKI), Wanfang Database and Chinese Scientific Journal Database (VIP).

\section{Searching other resources}

Clinical trials registry platforms will be searched, including the International Clinical Trials Registry platform (http://www.who.int/ictrp/network/primary/en/), the US National Institutes of Health Ongoing Trials Register (http://clinicaltrials.gov/), and the ISRCTN registry (http://www.controlled-trials.com/). We will also screen the reference lists of retrieved articles to identify missing eligible studies.

\section{Search strategies}

Search strategies in English electronic databases will be listed in online supplementary appendix 1 , and will be adapted for other resources with appropriate terms. No language restriction will be applied.

\section{Study selection}

Two reviewers will independently screen all titles and abstracts of the records. Full texts of potentially eligible studies will be retrieved for further identification according to the eligibility criteria. Any uncertainty or discrepancy will be resolved by discussion. Details of the study screening process will be shown as in figure 1 .

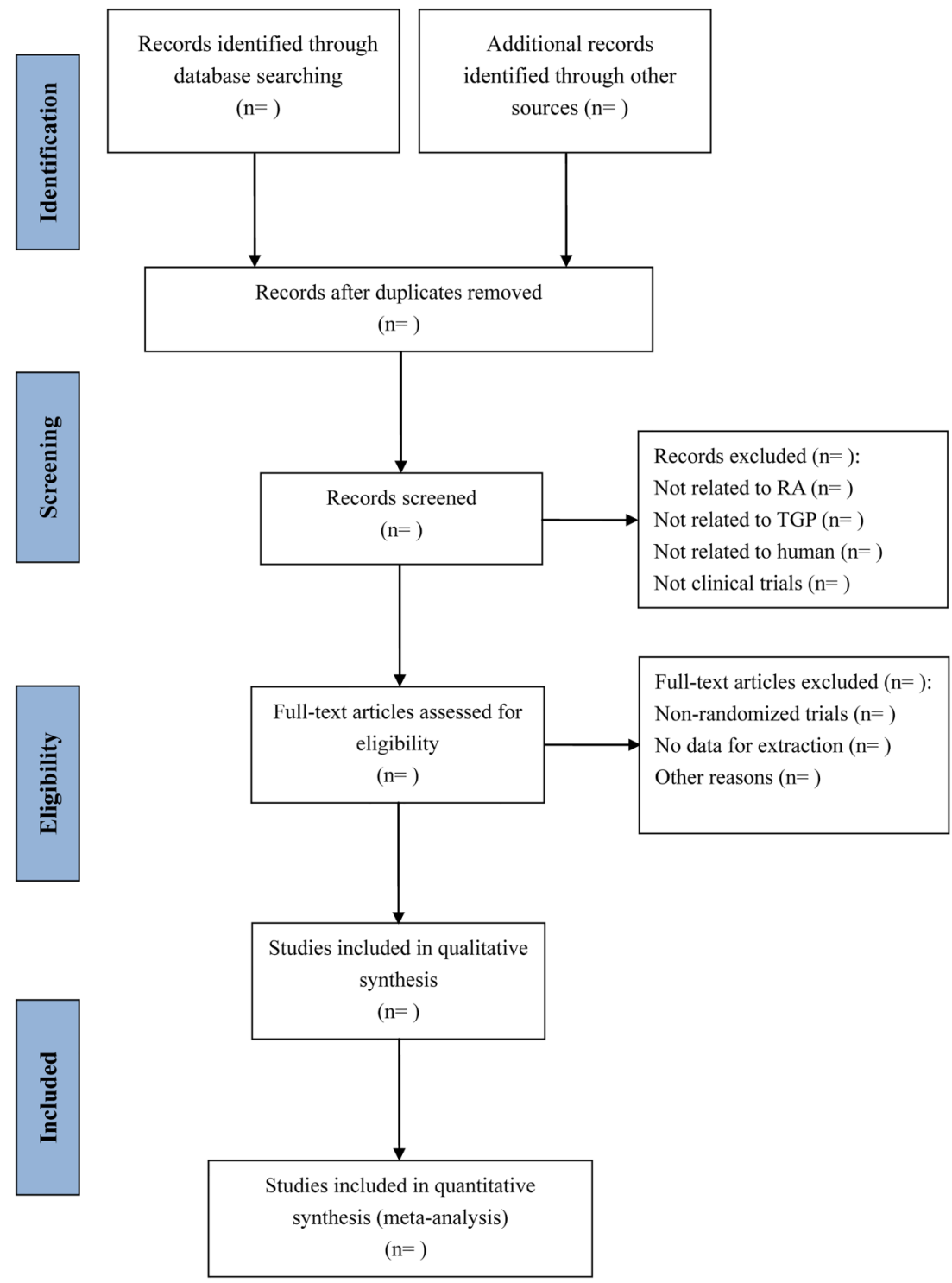

Figure 1 Flow diagram of study search and identification. RA, rheumatoid arthritis; TGP, total glucosides of paeony. 


\section{Data extraction}

Two reviewers will independently extract data in accordance with a predesigned data form using Excel (version Microsoft Excel 2007). Data will be checked by an additional two reviewers. Disagreements will be resolved by discussion.

Extracted information will comprise the following sections:

1. General information (publication years, number of authors, the first author, study design, sample size, demographics, setting)

2. Participants (diagnostic criteria, condition of RA, baseline comparison, withdrawals, loss to follow-up)

3. Interventions (dosage, administration, duration, follow-up, comparisons)

4. Outcome measures, results and adverse effects

We will seek missing information by contacting the original authors whenever possible. Any discrepancies will be resolved by discussion or consulting a third reviewer.

\section{Quality assessment}

\section{Assessment of risk of bias}

Two reviewers will independently evaluate the risk of bias for each included study using the Cochrane Collaboration's risk of bias tool, consisting of the following items: random sequence generation, allocation concealment, blinding of participants and personnel, blinding of outcome assessment, incomplete outcome data, selective reporting and other bias. ${ }^{25}$ We will judge each item as low, high or unclear risk of bias. Any uncertainty or discrepancy will be resolved by consulting a third reviewer.

\section{Quality of evidence}

We will judge the quality of evidence for the main comparison according to the Grading of Recommendations Assessment, Development and Evaluation (GRADE) method. ${ }^{26}$ The following five factors will be judged for each outcome in the main comparison: limitations in study design and execution, inconsistency of results, indirectness of evidence, imprecision, and publication bias. Accordingly, the quality of the evidence for each outcome will be graded as high, moderate, low or very low.

\section{Data analysis}

RevMan V.5.3 software will be used for data analysis. Studies included will be stratified by different types of comparison. Dichotomous data will be reported as risk ratios, and continuous data as mean difference or weighted mean difference, both with their 95\% CIs. We will perform intention-to-treat analysis where possible. We will attempt to obtain missing or incomplete data by contacting the original authors.

Meta-analyses will be performed if the data are sufficiently homogeneous, both statistically and clinically. Otherwise, analyses will be descriptive. Before pooling of data, heterogeneity will be tested using $\mathrm{I}^{2}$. If heterogeneity is low $\left(\mathrm{I}^{2} \leq 50 \%\right)$, a fixed-effects model will be applied to analyse data, and a random-effects model will be used when heterogeneity is moderate $\left(50 \%<\mathrm{I}^{2}<75 \%\right)$. Data will not be pooled when heterogeneity is high $\left(\mathrm{I}^{2} \geq 75 \%\right)$.

We will perform subgroup analyses according to different clinical characteristics (eg, different durations), and sensitivity analyses on the basis of study quality where possible. Funnel plots will be created to detect possible publication bias when sufficient studies (more than eight) are identified.

In addition, we will generate a 'Summary of findings' table using the GRADE profiler (V.3.6) to calculate the relative effect and the number of patients needed to treat in order to present important outcomes and the strength of evidence supporting these outcomes under the main comparison.

\section{Ethics and dissemination}

Ethics approval is not required, as this study will not involve patients. The results of this study will be submitted to a peer-reviewed journal for publication, to inform both clinical practice and further research.

\section{DISCUSSION}

RA can cause pain, joint destruction, and disability, placing a considerable burden on patients and society. Currently, DMARDs are the first-line treatment for RA. However, some patients have to stop treatment with DMARDs because of adverse effects, and some patients do not respond to DMARDs. Biological agents have been proven to be effective for RA, but their very high cost limits their use. In China, TGP, a natural plant extract, has been approved as a disease-modifying drug for RA since 1998 and is widely used for RA treatment. Some RCTs have reported beneficial effects of TGP in RA treatment, alone or in combination with other DMARDs. However, the effectiveness and safety of TGP for RA have not been systematically reviewed according to the PRISMA statement. This systematic review will provide a high-quality synthesis of current evidence for patients and rheumatologists seeking alternative and beneficial treatments of RA.

The strengths of this systematic review are twofold. First, this will be the first PRISMA-compliant systematic review to assess the effectiveness and safety of TGP for patients with RA. The study selection, data extraction and quality assessment will be conducted independently by two reviewers. Second, in addition to measuring methodological quality of each included study, this systematic review will evaluate the strength of evidence according to the GRADE approach. However, limitations may also exist. Although we will conduct an extensive and unbiased search, some unpublished RCTs with negative findings might be missed. We will detect possible publication bias through funnel plots in order to obtain an objective conclusion. In addition, it might be 
difficult to retrieve raw data from some published papers. We will try to contact the original authors to seek information. However, we believe the results of this study could provide objective evidence on the effects of TGP in RA treatment, which will be beneficial for patients and practitioners.

Contributors Q-WT and JL conceived and designed the study. JL and D-EJ developed the search strategy. G-YY and Q-WT provided methodological perspectives. JL drafted and refined the study protocol with contributions from all coauthors (G-YY, D-EJ, Y-ZZ, J-MW, W-PK and Q-WT). Y-ZZ and W-PK will search articles and conduct study selection. JL and J-MW will perform data extraction and assessment of quality. D-EJ will conduct data analyses. Y-ZZ and Q-WT will verify data extraction and data analyses. All authors read and approved the final manuscript.

Funding This work is supported by the Program of Beijing Natural Science Foundation (No 7132222).

Competing interests None declared.

Provenance and peer review Not commissioned; externally peer reviewed.

Data sharing statement All data regarding this protocol have been reported in this article.

Open Access This is an Open Access article distributed in accordance with the Creative Commons Attribution Non Commercial (CC BY-NC 4.0) license, which permits others to distribute, remix, adapt, build upon this work noncommercially, and license their derivative works on different terms, provided the original work is properly cited and the use is non-commercial. See: http:// creativecommons.org/licenses/by-nc/4.0/

\section{REFERENCES}

1. American College of Rheumatology. Rheumatoid Arthritis, 2013. http://www.rheumatology.org/l-Am-A/Patient-Caregiver/ Diseases-Conditions/Rheumatoid-Arthritis (accessed 28 Aug 2015).

2. Cross M, Smith E, Hoy D, et al. The global burden of rheumatoid arthritis: estimates from the Global Burden of Disease 2010 study. Ann Rheum Dis 2014;73:1316-22.

3. Curtis JR, Beukelman T, Onofrei A, et al. Elevated liver enzyme tests among patients with rheumatoid arthritis or psoriatic arthritis treated with methotrexate and/or leflunomide. Ann Rheum Dis 2010;69:43-7.

4. Lopez-Olivo MA, Amezaga Urruela M, McGahan L, et al. Rituximab for rheumatoid arthritis. Cochrane Database Syst Rev 2015;(1): CD007356.

5. Lethaby A, Lopez-Olivo MA, Maxwell L, et al. Etanercept for the treatment of rheumatoid arthritis. Cochrane Database Syst Rev 2013;(5):CD004525.

6. Singh JA, Cameron C, Noorbaloochi S, et al. Risk of serious infection in biological treatment of patients with rheumatoid arthritis: a systematic review and meta-analysis. Lancet 2015;386:258-65.

7. Ernst $\mathrm{E}$. Herbal medicine in the treatment of rheumatic diseases. Rheum Dis Clin North Am 2011;37:95-102.

8. Zhang W, Dai SM. Mechanisms involved in the therapeutic effects of Paeonia lactiflora Pallas in rheumatoid arthritis. Int Immunopharmacol 2012;14:27-31.
9. Chang $\mathrm{Y}$, Wei W, Zhang L, et al. Effects and mechanisms of total glucosides of paeony on synoviocytes activities in rat collagen-induced arthritis. J Ethnopharmacol 2009;121:43-8.

10. Zhao M, Liang GP, Tang MN, et al. Total glucosides of paeony induces regulatory CD4(+)CD25(+) T cells by increasing Foxp3 demethylation in lupus CD4(+) T cells. Clin Immunol 2012;143:180-7.

11. He DY, Dai SM. Anti-inflammatory and immunomodulatory effects of Paeonia lactiflora Pall., a traditional Chinese herbal medicine. Front Pharmacol 2011;2:10.

12. Min WQ, Wei Q, Li HY, et al. A clinical study of total glucosides of paeony in the treatment of rheumatoid arthritis: a multi-center trial. Chin J Rheumatol 2005;9:487-91.

13. Xiang N, Li XM, Zhang MJ, et al. Total glucosides of paeony can reduce the hepatotoxicity caused by Methotrexate and Leflunomide combination treatment of active rheumatoid arthritis. Int Immunopharmacol 2015;28:802-7.

14. Shang W, Guo JH, Cai H. Meta-analysis of total glucosides of paeony combined with methotrexate in treatment for rheumatoid arthritis. Mod J Integr Tradit Chin West Med 2010:19:653-6.

15. Li HC, Wen CP, Wang MJ, et al. Meta-Analysis of total glucosides of paeony combined with methorexate in treating rheumatoid arthritis. China J Tradit Chin Med Pharm 2012;27:1115-19.

16. Zhong $\mathrm{X}$, Su N, Zhou SH, et al. Meta-analysis of the efficacy and safety of total glucosides of paeony combined with immunosuppressant in the treatment of rheumatoid arthritis. China Pharm 2010;21:3731-4.

17. Guo XJ, Li S, Huang YS. Efficacy and safety of total glucosides of paeony for rheumatoid arthritis. J Jinggangshan Univ (Nat Sci) 2013;34:83-7.

18. Moher D, Liberati A, Tetzlaff J, et al. Preferred reporting items for systematic reviews and meta-analyses: the PRISMA statement. BMJ 2009;339:b2535.

19. Luo J, Jin DE, Yang GY, et al. Total glucosides of paeony for rheumatoid arthritis: a systematic review of randomized controlled trials. PROSPERO 2015:CRD42015026345. http://www.crd.york.ac. uk/PROSPERO_REBRANDING/display_record.asp? ID=CRD42015026345

20. Arnett FC, Edworthy SM, Bloch DA, et al. The American Rheumatism Association 1987 revised criteria for the classification of rheumatoid arthritis. Arthritis Rheum 1988;31:315-24.

21. Aletaha D, Neogi T, Silman AJ, et al. 2010 Rheumatoid arthritis classification criteria: an American College of Rheumatology/ European League Against Rheumatism collaborative initiative. Arthritis Rheum 2010;62:2569-81.

22. Felson DT, Anderson JJ, Boers M, et al. American College of Rheumatology. Preliminary definition of improvement in rheumatoid arthritis. Arthritis Rheum 1995;38:727-35.

23. Prevoo ML, van't Hof MA, Kuper $\mathrm{HH}$, et al. Modified disease activity scores that include twenty-eight-joint counts. Development and validation in a prospective longitudinal study of patients with rheumatoid arthritis. Arthritis Rheum 1995;38:44-8.

24. Fransen J, Creemers MC, Van Riel PL. Remission in rheumatoid arthritis: agreement of the disease activity score (DAS28) with the ARA preliminary remission criteria. Rheumatology (Oxford) 2004;43:1252-5.

25. Higgins JPT, Green S. Cochrane Handbook for Systematic Reviews of Interventions Version 5.1.0[updated March 2011]. The Cochrane Collaboration, 2011. http://www.cochrane-handbook.org

26. Schünemann $\mathrm{H}$, Brożek J, Oxman A. GRADE handbook for grading quality of evidence and strength of recommendation. Version 3.2 [updated March 2009]. The GRADE Working Group, 2009. http:// www.cc-ims.net/gradepro 